\title{
Reflected Backward Stochastic Differential Equations Driven by Countable Brownian Motions
}

\author{
Pengju Duan, ${ }^{1,2}$ Min Ren, $^{2}$ and Shilong Fei ${ }^{2}$ \\ ${ }^{1}$ Laboratory of Intelligent Information Processing, Suzhou University, Anhui 234000, China \\ ${ }^{2}$ Department of Mathematics, Suzhou University, Anhui 234000, China
}

Correspondence should be addressed to Pengju Duan; pjduan1981@hotmail.com

Received 16 June 2013; Accepted 15 September 2013

Academic Editor: Lotfollah Najjar

Copyright (C) 2013 Pengju Duan et al. This is an open access article distributed under the Creative Commons Attribution License, which permits unrestricted use, distribution, and reproduction in any medium, provided the original work is properly cited.

This paper deals with a new class of reflected backward stochastic differential equations driven by countable Brownian motions. The existence and uniqueness of the RBSDEs are obtained via Snell envelope and fixed point theorem.

\section{Introduction}

The nonlinear backward stochastic differential equations (BSDEs in short) were introduced by Pardoux and Peng [1], who proved the existence and uniqueness of the solution under the Lipschitz conditions for giving the probabilistic interpretation of semilinear parabolic partial differential equations. Since then, many authors were devoted to studying the BSDEs (see, e.g., [2-8] and the references therein). At present, the theory of BSDEs becomes a powerful tool to solve practical matters. In 1994, Pardoux and Peng [9] firstly studied the backward doubly stochastic differential equations (BDSDEs in short), which are driven by two kinds of Brownian motions. Later, Boufoussi et al. [10] established the connection between a class of generalized BDSDEs and semilinear stochastic partial differential equations with a Neumann boundary condition.

Reflected backward differential equations (RBSDEs in short) were introduced by El Karoui et al. [11]. Later, many researchers discussed various kinds of RBSDEs for their deep application in mathematical finance and partial differential equations. Ren and Hu [12] proposed the RBSDEs, driven by Teugels martingales and Brownian motion, and derived the existence and uniqueness of the solution by means of the Snell envelope and the fixed point theorem when the barrier was right continuous with left limits. Ren and El Otmani [13] discussed the generalized reflected BSDEs driven by Lévy process. Recently, Ren et al. [14] studied a new class of reflected backward doubly stochastic differential equations driven by Lévy process and Brownian motion.

As in all the previous works, the equations are driven by finite Brownian motions. To the best of our knowledge, there are no papers on the reflected backward stochastic differential equations driven by countable Brownian motions. In this paper, we aim to derive the existence and uniqueness of the solution for the RBSDEs driven by countable Brownian motions.

The structure of the paper is organized as follows. In Section 2, we give some notations. Section 3 is devoted to the main result.

\section{Notations}

Let $T$ be a positive constant. Throughout the paper $(\Omega, \mathscr{F}, \mathbb{P})$ is a complete probability space equipped with the natural filtration $\left\{\mathscr{F}_{t}\right\}_{t \geq 0}$ satisfying the usual conditions. $\left\{\beta_{j}(t)\right\}_{j=1}^{\infty}$ are mutual independent one-dimensional standard Brownian motions on the probability space. $W(t)$ is a standard Brownian motion on $\mathbb{R}^{d}$ which is independent of $\beta_{j}(t)$. Assume that

$$
\mathscr{F}_{t}=\left(\bigvee_{j=1}^{\infty} \mathscr{F}_{t, T}^{\beta_{j}}\right) \bigvee \mathscr{F}_{t}^{W} \bigvee \mathcal{N},
$$

where for any process $\left\{\eta_{t}\right\}, \mathscr{F}_{s, t}^{\eta}=\sigma\left\{\eta_{r}-\eta_{s}: s \leq r \leq t\right\}$, $\mathscr{F}_{t}^{\eta}=\mathscr{F}_{0, t}^{\eta}$, and $\mathcal{N}$ denotes the class of $\mathbb{P}$-null sets of $\mathscr{F}$. 
For the convenience, let us introduce some spaces:

(i) $\mathscr{H}^{2}=\left\{\left(\varphi_{t}\right)_{0 \leq t \leq T}\right.$ : an $\mathscr{F}_{t}$-progressively measurable, $\mathbb{R}$ valued process such that $\left.E \int_{0}^{T}\left|\varphi_{t}\right|^{2} \mathrm{~d} t<\infty\right\}$;

(ii) $\mathcal{S}^{2}=\left\{\left(\psi_{t}\right)_{0 \leq t \leq T}\right.$ : an $\mathscr{F}_{t}$-progressively measurable, $\mathbb{R}^{d}$-valued continuous process such that $\left.E\left(\sup _{0 \leq t \leq T}\left|\psi_{t}\right|^{2}\right)<\infty\right\} ;$

(iii) $\mathscr{A}^{2}=\left\{\left(K_{t}\right)_{0 \leq t \leq T}\right.$ : an $\mathscr{F}_{t}$-adapted, continuous, increasing process such that $\left.K_{0}=0, E\left|K_{t}\right|^{2}<\infty\right\}$.

With the previous preparations, we consider the following RBSDEs:

$$
\begin{aligned}
Y_{t}= & \xi+\int_{t}^{T} f\left(s, Y_{s}, Z_{s}\right) \mathrm{d} s \\
& +\sum_{j=1}^{\infty} \int_{t}^{T} g_{j}\left(s, Y_{s}, Z_{s}\right) \mathrm{d} \beta_{j}(s) \\
& -\int_{t}^{T} Z_{s} \mathrm{~d} W(s)+K_{T}-K_{t}, \quad 0 \leq t \leq T,
\end{aligned}
$$

where $f: \Omega \times[0, T] \times \mathbb{R} \times \mathbb{R}^{d} \rightarrow \mathbb{R}$ and $g_{j}: \Omega \times[0, T] \times$ $\mathbb{R} \times \mathbb{R}^{d} \rightarrow \mathbb{R}$

Definition 1. A solution of (2) is a triple of $\mathbb{R} \times \mathbb{R}^{d} \times \mathbb{R}_{+}$value process $\left(Y_{t}, Z_{t}, K_{t}\right)_{0 \leq t \leq T}$, which satisfies (2), and

(i) $\left(Y_{t}, Z_{t}, K_{t}\right)_{0 \leq t \leq T} \in \mathcal{S}^{2} \times \mathscr{H}^{2} \times \mathscr{A}^{2}$;

(ii) $Y_{t} \geq S_{t}$;

(iii) $K_{t}$ is a continuous and increasing process with $K_{0}=0$ and $\int_{0}^{T}\left(Y_{t}-S_{t}\right) \mathrm{d} K_{t}=0$

In order to get the solution of (2), we propose the following assumptions:

(H1) $\xi$ is an $\mathscr{F}_{T}$ measurable square integrable random variable;

(H2) the obstacle $\left\{S_{t}: 0 \leq t \leq T\right\}$ is an $\mathscr{F}_{t}$-progressive measurable continuous real valued process which satisfies $E \sup _{0 \leq t \leq T}\left(S_{t}\right)^{2}<\infty$. We always assume that $S_{T} \leq \xi$, a.s.;

(H3) $f(\cdot, y, z)$ and $g_{j}(\cdot, y, z)$ are two progressive measurable functions such that, for any $t \in[0, T], y_{1}, y_{2} \in \mathbb{R}$, $z_{1}, z_{2} \in \mathbb{R}^{d}$,

(3a) $f(s, \cdot, \cdot)$ is continuous and $|f(s, y, z)| \leq M(1+$ $|y|+|z|)$

(3b) $E \int_{0}^{T}|f(t, 0,0)|^{2} \mathrm{dt} \quad<\quad \infty$, $\sum_{j=1}^{\infty} E \int_{0}^{T}\left|g_{j}(t, 0,0)\right|^{2} \mathrm{dt}<\infty ;$

(3c) $\left|f\left(s, y_{1}, z_{1}\right)-f\left(s, y_{2}, z_{2}\right)\right|^{2} \leq C\left(\left|y_{1}-y_{2}\right|^{2}+\right.$ $\left.\left|z_{1}-z_{2}\right|^{2}\right), \quad\left|g_{j}\left(s, y_{1}, z_{1}\right)-g_{j}\left(s, y_{2}, z_{2}\right)\right|^{2} \leq$ $C_{j}\left|y_{1}-y_{2}\right|^{2}+\alpha_{j}\left|z_{1}-z_{2}\right|^{2}$, where $M, C$, $C_{j}$, and $\alpha_{j}$ are nonnegative constants with $\sum_{j=1}^{\infty} C_{j}<\infty$ and $\alpha=\sum_{j=1}^{\infty} \alpha_{j}<1$.

\section{Main Result}

In order to get the solution of (2), we consider the following RBSDEs driven by finite Brownian motions:

$$
\begin{aligned}
Y_{t}= & \xi+\int_{t}^{T} f\left(s, Y_{s}, Z_{s}\right) \mathrm{d} s \\
& +\sum_{j=1}^{n} \int_{t}^{T} g_{j}\left(s, Y_{s}, Z_{s}\right) \mathrm{d} \beta_{j}(s) \\
& -\int_{t}^{T} Z_{s} \mathrm{~d} W(s)+K_{T}-K_{t}, \quad 0 \leq t \leq T .
\end{aligned}
$$

Firstly, we consider a special case of (3); that is, the functions $f$ and $g$ do not depend on $(Y, Z)$ :

$$
\begin{aligned}
Y_{t}= & +\int_{t}^{T} f(s) \mathrm{d} s \\
& +\sum_{j=1}^{n} \int_{t}^{T} g_{j}(s) \mathrm{d} \beta_{j}(s)-\int_{t}^{T} Z_{s} \mathrm{~d} W(s) \\
& +K_{T}-K_{t}, \quad 0 \leq t \leq T, n \geq 1 .
\end{aligned}
$$

We will get the existence and uniqueness of the solution of (4) by means of Snell envelope and martingale representation theorem.

Theorem 2. Assume that (H1)-(H2), $f \in \mathscr{H}^{2}, g \in \mathscr{H}^{2}$. Then, there exists a triple $\left(Y_{t}, Z_{t}, K_{t}\right)_{0 \leq t \leq T} \in \mathcal{S}^{2} \times \mathscr{H}^{2} \times \mathscr{A}^{2}$ which is a solution of (4).

Proof. Let

$$
\mathscr{C}_{t}=\mathscr{F}_{t}^{W} \bigvee\left(\bigvee_{j=1}^{n} \mathscr{F}_{t, T}^{\beta_{j}}\right),
$$

and we define $\eta=\left\{\eta_{t}\right\}_{0 \leq t \leq T}$ as

$$
\begin{aligned}
\eta_{t}= & \xi 1_{\{t=T\}}+S_{t} 1_{\{t<T\}}+\int_{0}^{t} f(s) \mathrm{d} s \\
& +\sum_{j=1}^{n} \int_{0}^{t} g_{j}(s) \mathrm{d} \beta_{j}(s) .
\end{aligned}
$$

Then, $\eta$ is $\mathscr{C}_{t}$-adapted continuous process; furthermore;

$$
\sup _{0 \leq t \leq T}\left|\eta_{t}\right| \in L^{2}(\Omega)
$$

So, the Snell envelope of $\eta$ is given by

$$
S_{t}(\eta)=\underset{v \in \mathscr{T}}{\operatorname{ess} \sup } E\left[\eta_{\nu} \mid \mathscr{C}_{t}\right]
$$

where $\mathscr{T}$ is the set of all $\mathscr{C}_{t}$ stopping time such that $0 \leq v \leq T$. 
By the definition of $\eta$, we can deduce that

$$
E\left[\sup _{0 \leq t \leq T}\left|S_{t}(\eta)\right|^{2}\right]<\infty \text {. }
$$

Due to the Doob-Meyer decomposition, we have

$$
\begin{aligned}
S_{t}(\eta)=E[\xi & +\int_{0}^{T} f(s) \mathrm{d} s \\
& \left.+\sum_{j=1}^{n} \int_{0}^{T} g_{j}(s) \mathrm{d} \beta_{j}(s)+K_{T} \mid \mathscr{C}_{t}\right]-K_{t},
\end{aligned}
$$

where $\left\{K_{t}\right\}_{0 \leq t \leq T}$ is a $\mathscr{C}_{t}$-adapted, continuous, and nondecreasing process such that $K_{0}=0$ and $E K_{T}^{2}<\infty$. So, we have

$$
\begin{aligned}
E\left[\sup _{0 \leq t \leq T} \mid E[\xi\right. & +\int_{0}^{T} f(s) \mathrm{d} s \\
& \left.\left.+\sum_{j=1}^{n} \int_{0}^{T} g_{j}(s) \mathrm{d} \beta_{j}(s)+K_{T} \mid \mathscr{C}_{t}\right]\left.\right|^{2}\right]<\infty
\end{aligned}
$$

Martingale representation theorem yields that there exists $\mathscr{C}_{t}$-progressive measurable process $\left\{Z_{t}\right\} \in \mathbb{R}^{d}$ such that

$$
\begin{aligned}
M_{t} & \triangleq E\left[\xi+\int_{0}^{T} f(s) \mathrm{d} s+\sum_{j=1}^{n} \int_{0}^{T} g_{j}(s) \mathrm{d} \beta_{j}(s)+K_{T} \mid \mathscr{C}_{t}\right] \\
& =M_{0}+\int_{0}^{t} Z_{s} \mathrm{~d} W(s), \quad 0 \leq t \leq T .
\end{aligned}
$$

Let $Y_{t}=\operatorname{ess} \sup _{\nu \in \mathscr{T}} E\left[\xi 1_{\{v=T\}}+S_{\nu} 1_{\{v<T\}}+\int_{t}^{v} f(s) \mathrm{d} s+\right.$ $\left.\sum_{j=1}^{n} \int_{t}^{v} g_{j}(s) \mathrm{d} \beta_{j}(s) \mid \mathscr{C}_{t}\right]$; then,

$$
\begin{aligned}
Y_{t} & +\int_{0}^{t} f(s) \mathrm{d} s+\sum_{j=1}^{n} \int_{0}^{t} g_{j}(s) \mathrm{d} \beta_{j}(s) \\
& =S_{t}(\eta)=M_{t}-K_{t} \\
& =M_{0}+\int_{0}^{t} Z_{s} \mathrm{~d} W(s)-K_{t}, \quad 0 \leq t \leq T .
\end{aligned}
$$

Therefore,

$$
\begin{aligned}
Y_{t}= & \xi+\int_{t}^{T} f(s) \mathrm{d} s+\sum_{j=1}^{n} \int_{t}^{T} g_{j}(s) \mathrm{d} \beta_{j}(s) \\
& -\int_{t}^{T} Z_{s} \mathrm{~d} W(s)+K_{T}-K_{t} .
\end{aligned}
$$

By the definitions of $Y_{t}$ and $S_{t}(\eta), \xi \geq S_{T}$,

$$
\begin{aligned}
Y_{t}+ & \int_{0}^{t} f(s) \mathrm{d} s+\sum_{j=1}^{n} \int_{0}^{t} g_{j}(s) \mathrm{d} \beta_{j}(s) \\
= & S_{t}(\eta) \geq \eta_{t} \\
= & \xi 1_{\{t=T\}}+S_{t} 1_{\{t<T\}}+\int_{0}^{t} f(s) \mathrm{d} s \\
& +\sum_{j=1}^{n} \int_{0}^{t} g_{j}(s) \mathrm{d} \beta_{j}(s) \\
\geq & S_{T} 1_{\{t=T\}}+S_{t} 1_{\{t<T\}}+\int_{0}^{t} f(s) \mathrm{d} s \\
& +\sum_{j=1}^{n} \int_{0}^{t} g_{j}(s) \mathrm{d} \beta_{j}(s) .
\end{aligned}
$$

So, we have $Y_{t} \geq S_{t}$.

Finally, from Hamadène [15], we get $\int_{0}^{T}\left(S_{t}(\eta)-\eta_{t}\right) \mathrm{d} K_{t}=0$; that is,

$$
\int_{0}^{T}\left(Y_{t}-S_{t}\right) \mathrm{d} K_{t}=0
$$

It shows that the process $\left(Y_{t}, Z_{t}, K_{t}\right)_{0 \leq t \leq T}$ is a solution of (4).

Theorem 3. Under the assumptions of (H1)-(H3), there exists a unique solution $\left(Y_{t}, Z_{t}, K_{t}\right)_{0 \leq t \leq T}$ of (3).

Proof. Let $\mathscr{P}=\mathcal{S}^{2} \times \mathscr{H}^{2}$ be endowed with the norm

$$
\|(Y, Z)\|_{\beta}=\left(E\left[\int_{0}^{T} e^{\beta s}\left(\left|Y_{s}\right|^{2}+\left|Z_{s}\right|^{2}\right) \mathrm{d} s\right]\right)^{1 / 2}
$$

for a suitable constant $\beta>0$. We define the map $\Phi$ from $\mathscr{P}$ into itself and $(\widetilde{Y}, \widetilde{Z})$ and $\left(\widetilde{Y^{\prime}}, \widetilde{Z^{\prime}}\right)$ are two elements of $\mathscr{P}$. Define $(Y, Z)=\Phi(\widetilde{Y}, \widetilde{Z}),\left(Y^{\prime}, Z^{\prime}\right)=$ $\Phi\left(\widetilde{Y^{\prime}}, \widetilde{Z^{\prime}}\right)$, where $(Y, Z, K)$ and $\left(Y^{\prime}, Z^{\prime}, K^{\prime}\right)$ are solutions of $(4)$ 
associated with $\left(\xi, f(t, \widetilde{Y}, \widetilde{Z}), g_{j}(t, \widetilde{Y}, \widetilde{Z}), S\right)$, and $\left(\xi, f\left(t, \widetilde{Y^{\prime}}\right.\right.$, $\left.\left.\widetilde{Z^{\prime}}\right), g_{j}\left(t, \widetilde{Y^{\prime}}, \widetilde{Z^{\prime}}\right), S^{\prime}\right)$, respectively. Set $(\bar{Y}, \bar{Z})=\left(Y_{t}-Y_{t}^{\prime}, Z_{t}-\right.$ $Z_{t}^{\prime}$ ) and

$$
\begin{aligned}
\Psi_{M}(x)= & x^{2} \mathbf{1}_{\{-M \leq x \leq M\}}+M(2 x-M) \mathbf{1}_{\{x>M\}} \\
& -M(2 x+M) \mathbf{1}_{\{x<-M\}} .
\end{aligned}
$$

If we define $\Psi_{M}^{\prime}(x) / x=2$, when $x=0$, then, $0 \leq \Psi_{M}^{\prime}\left(\overline{Y_{s}}\right) / \overline{Y_{s}} \leq$ 2. Applying Itô formula to $e^{\beta t} \Psi_{M}\left(\overline{Y_{s}}\right)$, we have

$$
\begin{aligned}
& e^{\beta t} \Psi_{M}\left(\overline{Y_{t}}\right)+\beta \int_{t}^{T} e^{\beta s} \Psi_{M}\left(\overline{Y_{s}}\right) \mathrm{d} s \\
& \quad+\int_{t}^{T} e^{\beta s} \mathbf{1}_{\left\{-M \leq \overline{Y_{s}} \leq M\right\}}\left|\overline{Z_{s}}\right|^{2} \mathrm{~d} s \\
& =\int_{t}^{T} e^{\beta s} \Psi_{M}^{\prime}\left(\overline{Y_{s}}\right)\left(f\left(s, \widetilde{Y_{s}}, \widetilde{Z_{s}}\right)-f\left(s, \widetilde{Y_{s}^{\prime}}, \widetilde{Z_{s}^{\prime}}\right)\right) \mathrm{d} s \\
& +\sum_{j=1}^{n} \int_{t}^{T} e^{\beta s} \mathbf{1}_{\left\{-M \leq \overline{Y_{s}} \leq M\right\}} \mid g_{j}\left(s, \widetilde{Y_{s}}, \widetilde{Z_{s}}\right) \\
& \quad-\sum_{j=1}^{n} \int_{t}^{T} e^{\beta s} \Psi_{M}^{\prime}\left(\overline{Y_{s}}\right)\left(g_{j}\left(s, \widetilde{Y_{s}}, \widetilde{Z_{s}}\right)\right. \\
& \left.\quad-\int_{t}^{T}, \widetilde{Z_{s}^{\prime}}\right)\left.\right|^{2} \mathrm{~d} s \\
& \left.\quad+\int_{t}^{T} e^{\beta s} \Psi_{M}^{\prime}\left(s, \widetilde{Y_{s}^{\prime}}, \widetilde{Z_{s}^{\prime}}\right)\right) \mathrm{d} \beta_{j}(s) \\
& \left.\quad \overline{Y_{s}}\right) \overline{Y_{s}} \mathrm{~d} W(s)\left(\mathrm{d} K_{s}-\mathrm{d} K_{s}^{\prime}\right) .
\end{aligned}
$$

Taking expectation on both sides of (19) and noticing that $\int_{t}^{T} e^{\beta s} \Psi_{M}^{\prime}\left(\overline{Y_{s}}\right)\left(\mathrm{d} K_{s}-\mathrm{d} K_{s}^{\prime}\right) \leq 0$, we have

$$
\begin{aligned}
E e^{\beta t} \Psi_{M}\left(\overline{Y_{t}}\right)+E \beta \int_{t}^{T} e^{\beta s} \Psi_{M}\left(\overline{Y_{s}}\right) \mathrm{d} s & \\
& +E \int_{t}^{T} e^{\beta s} \mathbf{1}_{\left\{-M \leq \overline{Y_{s}} \leq M\right\}}\left|\overline{Z_{s}}\right|^{2} \mathrm{~d} s \\
\leq & E \int_{t}^{T} e^{\beta s} \Psi_{M}^{\prime}\left(\overline{Y_{s}}\right)\left(f\left(s, \widetilde{Y_{s}}, \widetilde{Z_{s}}\right)-f\left(s, \widetilde{Y_{s}^{\prime}}, \widetilde{Z_{s}^{\prime}}\right)\right) \mathrm{d} s \\
& +\sum_{j=1}^{n} E \int_{t}^{T} e^{\beta s} \mathbf{1}_{\left\{-M \leq \overline{Y_{s}} \leq M\right\}} \mid g_{j}\left(s, \widetilde{Y_{s}}, \widetilde{Z_{s}}\right) \\
& -\left.g_{j}\left(s, \widetilde{Y_{s}^{\prime}}, \widetilde{Z_{s}^{\prime}}\right)\right|^{2} \mathrm{~d} s
\end{aligned}
$$

$$
\begin{aligned}
\leq & 2 E \int_{t}^{T} e^{\beta s} \overline{Y_{s}}\left(f\left(s, \widetilde{Y_{s}}, \widetilde{Z_{s}}\right)-f\left(s, \widetilde{Y_{s}^{\prime}}, \widetilde{Z_{s}^{\prime}}\right)\right) \mathrm{d} s \\
& +\sum_{j=1}^{n} E \int_{t}^{T} e^{\beta s}\left|g_{j}\left(s, \widetilde{Y_{s}}, \widetilde{Z_{s}}\right)-g_{j}\left(s, \widetilde{Y_{s}^{\prime}}, \widetilde{Z_{s}^{\prime}}\right)\right|^{2} \mathrm{~d} s \\
\leq & \frac{2 C}{1-\alpha} E \int_{t}^{T} e^{\beta s}\left|\overline{Y_{s}}\right|^{2} \mathrm{~d} s \\
& +\left(\sum_{j=1}^{\infty} C_{j}+\frac{1-\alpha}{2}\right) E \int_{t}^{T} e^{\beta s}\left|\widetilde{Y_{s}}-\widetilde{Y_{s}^{\prime}}\right|^{2} \mathrm{~d} s \\
& +\frac{1+\alpha}{2} E \int_{t}^{T} e^{\beta s}\left|\widetilde{Z_{s}}-\widetilde{Z_{s}^{\prime}}\right|^{2} \mathrm{~d} s .
\end{aligned}
$$

Let $\gamma=2 C /(1-\alpha), \bar{C}=2\left(\sum_{j=1}^{\infty} C_{j}+((1-\alpha) / 2)\right) /(1+\alpha)$, $\beta=\gamma+\bar{C}$, and $M \rightarrow \infty$; we have

$$
\begin{gathered}
\bar{C} E \int_{t}^{T} e^{\beta s}\left|Y_{s}-Y_{s}^{\prime}\right|^{2} \mathrm{~d} s+E \int_{t}^{T} e^{\beta s}\left|Z_{s}-Z_{s}^{\prime}\right|^{2} \mathrm{~d} s \\
\leq \frac{1+\alpha}{2} E \int_{t}^{T} e^{\beta s}\left(\bar{C}\left|\widetilde{Y_{s}}-\widetilde{Y_{s}^{\prime}}\right|^{2}+\left|\widetilde{Z_{s}}-\widetilde{Z_{s}^{\prime}}\right|^{2}\right) ;
\end{gathered}
$$

that is,

$$
\left\|\left(Y_{s}, Z_{s}\right)\right\|_{\beta}^{2} \leq \frac{1+\alpha}{2}\left\|\left(Y_{s}^{\prime}, Z_{s}^{\prime}\right)\right\|_{\beta}^{2} .
$$

It follows that $\Phi$ is a strict contraction on $\mathscr{P}$ with the norm $\|\cdot\|_{\beta}$, where $\beta$ is defined as above. Then, $\Phi$ has a fixed point $(Y, Z, K)$ which is the unique solution of (4) from the Burkholder-Davis-Gundy inequality.

With all the preparations, we will give the main result of this paper as follows.

Theorem 4. Under the conditions of (H1)-(H3), there exists a unique solution $\left(Y_{t}, Z_{t}, K_{t}\right)_{0 \leq t \leq T} \in \mathcal{S}^{2} \times \mathscr{H}^{2} \times \mathscr{A}^{2}$ of (2).

Proof (existence). By Theorem 3, for any $n \geq 1$, there exists a unique solution of (3), denoted by $\left(Y_{t}^{n}, Z_{t}^{n}, K_{t}^{n}\right)$,

$$
\begin{aligned}
Y_{t}^{n}= & \xi+\int_{t}^{T} f\left(s, Y_{s}^{n}, Z_{s}^{n}\right) \mathrm{d} s \\
& +\sum_{j=1}^{n} \int_{t}^{T} g_{j}\left(s, Y_{s}^{n}, Z_{s}^{n}\right) \mathrm{d} \beta_{j}(s) \\
& -\int_{t}^{T} Z_{s}^{n} \mathrm{~d} W(s)+K_{T}^{n}-K_{t}^{n} .
\end{aligned}
$$

In the following parts, we will claim that $\left(Y_{t}^{n}, Z_{t}^{n}, K_{t}^{n}\right)$ is a Cauchy sequence in $\mathcal{S}^{2} \times \mathscr{H}^{2} \times \mathscr{A}^{2}$. Without loss of generality, 
we let $n<m$. Applying general Itô formula to $\left|Y_{t}^{n}-Y_{t}^{m}\right|^{2}$, we have.

$$
\begin{aligned}
& \left|Y_{t}^{n}-Y_{t}^{m}\right|^{2}+\int_{t}^{T}\left|Z_{s}^{n}-Z_{s}^{m}\right|^{2} \mathrm{~d} s \\
& =2 \int_{t}^{T}\left(Y_{s}^{n}-Y_{s}^{m}\right)\left(f\left(s, Y_{s}^{n}, Z_{s}^{n}\right)\right. \\
& \left.\quad-f\left(s, Y_{s}^{m}, Z_{s}^{m}\right)\right) \mathrm{d} s \\
& \quad+\sum_{j=n+1}^{m} \int_{t}^{T}\left|g_{j}\left(s, Y_{s}^{n}, Z_{s}^{n}\right)-g_{j}\left(s, Y_{s}^{m}, Z_{s}^{m}\right)\right|^{2} \mathrm{~d} s \\
& \quad-2 \sum_{j=n+1}^{m} \int_{t}^{T}\left(Y_{s}^{n}-Y_{s}^{m}\right)\left(g_{j}\left(s, Y_{s}^{n}, Z_{s}^{n}\right)\right. \\
& \quad-2 \int_{t}^{T}\left(Y_{s}^{n}-Y_{s}^{m}\right)\left(Z_{s}^{n}-Z_{s}^{m}\right) \mathrm{d} W(s) \\
& \quad+2 \int_{t}^{T}\left(Y_{s}^{n}-Y_{s}^{m}\right)\left(\mathrm{d} K_{s}^{n}-\mathrm{d} K_{s}^{m}\right) .
\end{aligned}
$$

Taking expectation on both sides of (24) and noting that $\int_{t}^{T}\left(Y_{s}^{n}-Y_{s}^{m}\right)\left(\mathrm{d} K_{s}^{n}-\mathrm{d} K_{s}^{m}\right) \leq 0$, we obtain

$$
\begin{aligned}
& E\left|Y_{t}^{n}-Y_{t}^{m}\right|^{2}+E \int_{t}^{T}\left|Z_{s}^{n}-Z_{s}^{m}\right|^{2} \mathrm{~d} s \\
& \leq 2 E \int_{t}^{T}\left(Y_{s}^{n}-Y_{s}^{m}\right)\left(f\left(s, Y_{s}^{n}, Z_{s}^{n}\right)\right. \\
& \left.\quad-f\left(s, Y_{s}^{m}, Z_{s}^{m}\right)\right) \mathrm{d} s \\
& +\sum_{j=n+1}^{m} E \int_{t}^{T}\left|g_{j}\left(s, Y_{s}^{n}, Z_{s}^{n}\right)-g_{j}\left(s, Y_{s}^{m}, Z_{s}^{m}\right)\right|^{2} \mathrm{~d} s .
\end{aligned}
$$

By (H3) and elementary inequality $2 a b \leq \beta a^{2}+(1 / \beta) b^{2}, \beta>$ 0 , we obtain

$$
\begin{aligned}
E \mid Y_{t}^{n} & -\left.Y_{t}^{m}\right|^{2}+E \int_{t}^{T}\left|Z_{s}^{n}-Z_{s}^{m}\right|^{2} \mathrm{~d} s \\
\leq & \frac{2 C}{1-\alpha} E \int_{t}^{T}\left|Y_{s}^{n}-Y_{s}^{m}\right|^{2} \mathrm{~d} s+\frac{1-\alpha}{2} E \int_{t}^{T}\left|Y_{s}^{n}-Y_{s}^{m}\right|^{2} \mathrm{~d} s \\
& +\frac{1-\alpha}{2} E \int_{t}^{T}\left|Z_{s}^{n}-Z_{s}^{m}\right|^{2} \mathrm{~d} s+\alpha E \int_{t}^{T}\left|Z_{s}^{n}-Z_{s}^{m}\right|^{2} \mathrm{~d} s \\
& +\left[\sum_{j=n+1}^{m} C_{j}\right] E \int_{t}^{T}\left|Y_{s}^{n}-Y_{s}^{m}\right|^{2} \mathrm{~d} s .
\end{aligned}
$$

Furthermore,

$$
\begin{gathered}
E\left|Y_{t}^{n}-Y_{t}^{m}\right|^{2}+\frac{1-\alpha}{2} E \int_{t}^{T}\left|Z_{s}^{n}-Z_{s}^{m}\right|^{2} \mathrm{~d} s \\
\leq C_{p} E \int_{t}^{T}\left|Y_{s}^{n}-Y_{s}^{m}\right|^{2} \mathrm{~d} s,
\end{gathered}
$$

where $C_{p}=(2 C /(1-\alpha))+((1-\alpha) / 2)+\sum_{j=n+1}^{m} C_{j}$.

By Gronwall's inequality and Burkholder-Davis-Gundy inequality, we have

$$
E\left[\sup _{0 \leq t \leq T} \int_{t}^{T}\left|Y_{s}^{n}-Y_{s}^{m}\right|^{2} \mathrm{~d} s\right] \longrightarrow 0 .
$$

Denote the limit of $\left(Y_{t}^{n}, Z_{t}^{n}, K_{t}^{n}\right)$ by $\left(Y_{t}, Z_{t}, K_{t}\right)$; we will show that $\left(Y_{t}, Z_{t}, K_{t}\right)$ satisfies (2). If it is necessary, we can choose a subsequence of (3). By Hölder's inequality,

$$
\begin{aligned}
& E\left|\int_{t}^{T}\left(f\left(s, Y_{s}, Z_{s}\right)-f\left(s, Y_{s}^{n}, Z_{s}^{n}\right)\right) \mathrm{d} s\right|^{2} \\
& \quad \leq T E \int_{t}^{T}\left|\left(f\left(s, Y_{s}, Z_{s}\right)-f\left(s, Y_{s}^{n}, Z_{s}^{n}\right)\right)\right|^{2} \mathrm{~d} s \longrightarrow 0 .
\end{aligned}
$$

From (27), we know

$$
E \int_{0}^{T}\left|Y_{t}^{n}-Y_{t}\right|^{2} \mathrm{~d} t \longrightarrow 0,
$$

and $Y_{t}^{n} \rightarrow Y_{t}$, a.e., so

$$
\sqrt{E \int_{0}^{T}\left|Y_{t}^{n+1}-Y_{t}^{n}\right|^{2} \mathrm{~d} t} \leq \frac{1}{2^{n}}
$$

For any $n$,

$$
\left|Y_{t}^{n}\right| \leq\left|Y_{t}^{1}\right|+\sum_{i=1}^{n-1}\left|Y_{t}^{i+1}-Y_{t}^{i}\right| \leq\left|Y_{t}^{1}\right|+\sum_{i=1}^{\infty}\left|Y_{t}^{i+1}-Y_{t}^{i}\right| .
$$

Then, we have

$$
\begin{aligned}
& \sqrt{E \int_{0}^{T} \sup _{n}\left|Y_{t}^{n}\right|^{2} \mathrm{~d} t} \\
& \leq \sqrt{E \int_{0}^{T}\left(\left|Y_{t}^{1}\right|+\sum_{i=1}^{\infty}\left|Y_{t}^{i+1}-Y_{t}^{i}\right|\right)^{2} \mathrm{~d} t} \\
& \leq \sqrt{E \int_{0}^{T}\left|Y_{t}^{1}\right|^{2} \mathrm{~d} t}+\sum_{i=1}^{\infty} \sqrt{E \int_{0}^{T}\left|Y_{t}^{i+1}-Y_{t}^{i}\right|^{2} \mathrm{~d} t} \\
& \leq \sqrt{E \int_{0}^{T}\left|Y_{t}^{1}\right|^{2} \mathrm{~d} t}+\sum_{i=1}^{\infty} \frac{1}{2^{i}} .
\end{aligned}
$$

From (H4), it follows

$$
\begin{aligned}
& E \int_{0}^{T} \sup _{n}\left|f\left(s, Y_{s}, Z_{s}\right)-f\left(s, Y_{s}^{n}, Z_{s}^{n}\right)\right|^{2} \mathrm{~d} s \\
& \quad \leq 2 C E \int_{0}^{T}\left(\sup _{n}\left|Y_{s}^{n}\right|^{2}+\left|Y_{s}\right|^{2}+\sup _{n}\left|Z_{s}^{n}\right|^{2}+\left|Z_{s}\right|^{2}\right) \mathrm{d} s<\infty .
\end{aligned}
$$


Applying Lebesgue dominated convergence theorem, we deduce that $\left(Y_{t}, Z_{t}, K_{t}\right)$ is the solution of (2) by continuity of the functions $f$ and $g$.

Uniqueness. Let $\left(Y_{t}^{i}, Z_{t}^{i}, K_{t}^{i}\right)(i=1,2)$ be two solutions of (2), $\bar{Y}_{t}=Y_{t}^{1}-Y_{t}^{2}, \bar{Z}_{t}=Z_{t}^{1}-Z_{t}^{2}$. We apply Itô formula to $e^{\beta t} \Psi_{M}\left(\bar{Y}_{t}\right)$, for any $\beta \in \mathbb{R}$,

$$
\begin{aligned}
e^{\beta t} \Psi_{M} & \left(\bar{Y}_{t}\right)+\beta \int_{t}^{T} e^{\beta s} \Psi_{M}\left(\bar{Y}_{s}\right) \mathrm{d} s \\
& +\int_{t}^{T} e^{\beta s} \mathbf{1}_{\left\{-M \leq \bar{Y}_{s} \leq M\right\}}\left|\bar{Z}_{s}\right|^{2} \mathrm{~d} s \\
= & \int_{t}^{T} e^{\beta s} \Psi_{M}^{\prime}\left(\bar{Y}_{s}\right)\left(f\left(s, Y_{s}^{1}, Z_{s}^{1}\right)-f\left(s, Y_{s}^{2}, Z_{s}^{2}\right)\right) \mathrm{d} s \\
& +\sum_{j=1}^{\infty} \int_{t}^{T} e^{\beta s} \mathbf{1}_{\left\{-M \leq \bar{Y}_{s} \leq M\right\}} \mid g_{j}\left(s, Y_{s}^{1}, Z_{s}^{1}\right) \\
& -\sum_{j=1}^{\infty} \int_{t}^{T} e^{\beta s} \Psi_{M}^{\prime}\left(\bar{Y}_{s}\right) \\
& +\int_{t}^{T} e^{\beta s} \Psi_{M}^{\prime}\left(\bar{Y}_{s}\right) \bar{Z}_{s} \mathrm{~d} W_{s} \\
& +\int_{j}^{T} e^{\beta s} \Psi_{M}^{\prime}\left(\bar{Y}_{s}\right)\left(\mathrm{d} K_{s}^{1}-\mathrm{d} K_{s}^{2}\right) . \\
& \times\left(g_{j}\left(s, Y_{s}^{2}, Z_{s}^{1}\right)-g_{j}\left(s, Y_{s}^{2}, Z_{s}^{2}\right)\right) \mathrm{d} \beta_{j}(s)
\end{aligned}
$$

Taking expectation on both sides of (35),

$$
\begin{aligned}
E e^{\beta t} \Psi_{M}\left(\bar{Y}_{t}\right)+\beta E \int_{t}^{T} e^{\beta s} \Psi_{M}\left(\bar{Y}_{s}\right) \mathrm{d} s & \\
& +E \int_{t}^{T} e^{\beta s} \mathbf{1}_{\left\{-M \leq \bar{Y}_{s} \leq M\right\}}\left|\bar{Z}_{s}\right|^{2} \mathrm{~d} s \\
\leq & 2 E \int_{t}^{T} e^{\beta s} \bar{Y}_{s}\left(f\left(s, Y_{s}^{1}, Z_{s}^{1}\right)-f\left(s, Y_{s}^{2}, Z_{s}^{2}\right)\right) \mathrm{d} s \\
& +\sum_{j=1}^{\infty} E \int_{t}^{T} e^{\beta s} \mathbf{1}_{\left\{-M \leq \bar{Y}_{s} \leq M\right\}} \mid g_{j}\left(s, Y_{s}^{1}, Z_{s}^{1}\right) \\
\leq & \left(\frac{2 C}{1-\sum_{j=1}^{\infty} \alpha_{j}}+\sum_{j=1}^{\infty} C_{j}+\frac{1-\sum_{j=1}^{\infty} \alpha_{j}}{2}\right) E \int_{t}^{T} e^{\beta s}\left|\bar{Y}_{s}\right|^{2} \mathrm{~d} s \\
& +\frac{1+\sum_{j=1}^{\infty} \alpha_{j}}{2} E \int_{t}^{T} e^{\beta s}\left|\bar{Z}_{t}\right|^{2} \mathrm{~d} s .
\end{aligned}
$$

Let $M \rightarrow \infty$, and applying monotone convergence theorem, we have

$$
\begin{gathered}
E e^{\beta t}\left|\bar{Y}_{t}\right|^{2}+\left(\beta-\frac{2 C}{1-\alpha}-\sum_{j=1}^{\infty} C_{j}-\frac{1-\alpha}{2}\right) \\
\times E \int_{t}^{T} e^{\beta s}\left|\bar{Y}_{s}\right|^{2} \mathrm{~d} s \\
+\frac{1-\alpha}{2} E \int_{t}^{T} e^{\beta s}\left|\bar{Z}_{s}\right|^{2} \mathrm{~d} s \leq 0 .
\end{gathered}
$$

When $\beta$ is taken sufficiently large, we have $\bar{Y}_{t}=0$, a.e., for all $s \in[t, T]$. So, we have $\bar{Z}_{t}=0$, a.e. Then, we complete the proof.

\section{Acknowledgments}

The authors would like to take this chance to express their sincere gratitude to the National Natural Science Foundation of China (11201004 and 11371029), Natural Science Foundation of Anhui Province (KJ2011B176 and KJ2013B288), Professors(Doctors) Scientific Research Foundation of Suzhou University (2013jb04), and Foundation of Laboratory of Intelligent Information Processing of Suzhou University (2010YKF11).

\section{References}

[1] E. Pardoux and S. G. Peng, "Adapted solution of a backward stochastic differential equation," Systems \& Control Letters, vol. 14, no. 1, pp. 55-61, 1990.

[2] X. Mao, "Adapted solutions of backward stochastic differential equations with non-Lipschitz coefficients," Stochastic Processes and their Applications, vol. 58, no. 2, pp. 281-292, 1995.

[3] K. Bahlali, E. H. Essaky, M. Hassani, and E. Pardoux, "Existence, uniqueness and stability of backward stochastic differential equations with locally monotone coefficient," Comptes Rendus Mathématique, vol. 335, no. 9, pp. 757-762, 2002.

[4] P. Briand, B. Delyon, Y. Hu, E. Pardoux, and L. Stoica, " $L^{p}$ solutions of backward stochastic differential equations," Stochastic Processes and their Applications, vol. 108, no. 1, pp. 109-129, 2003.

[5] J. P. Lepeltier and J. S. Martin, "Backward stochastic differential equations with continuous coefficient," Statistics \& Probability Letters, vol. 14, pp. 55-514, 1997.

[6] G. Jia, "A class of backward stochastic differential equations with discontinuous coefficients," Statistics \& Probability Letters, vol. 78, no. 3, pp. 231-237, 2008.

[7] N. El Karoui, S. Peng, and M. C. Quenez, "Backward stochastic differential equations in finance," Mathematical Finance, vol. 7, no. 1, pp. 1-71, 1997.

[8] S. Hamadene and J.-P. Lepeltier, "Zero-sum stochastic differential games and backward equations," Systems \& Control Letters, vol. 24, no. 4, pp. 259-263, 1995.

[9] E. Pardoux and S. G. Peng, "Backward doubly stochastic differential equations and systems of quasilinear SPDEs," Probability Theory and Related Fields, vol. 98, no. 2, pp. 209-227, 1994.

[10] B. Boufoussi, J. Casteren, and N. Mrhardy, "Generalized backward doubly stochastic differential equations and SPDEs with 
nonlinear Neumann boundary conditions," Bernoulli, vol. 13, no. 2, pp. 423-446, 2007.

[11] N. El Karoui, C. Kapoudjian, E. Pardoux, S. Peng, and M. C. Quenez, "Reflected solutions of backward SDE's, and related obstacle problems for PDE's," The Annals of Probability, vol. 25, no. 2, pp. 702-737, 1997.

[12] Y. Ren and L. Hu, "Reflected backward stochastic differential equations driven by Lévy processes," Statistics \& Probability Letters, vol. 77, no. 15, pp. 1559-1566, 2007.

[13] Y. Ren and M. El Otmani, "Generalized reflected BSDEs driven by a Lévy process and an obstacle problem for PDIEs with a nonlinear Neumann boundary condition," Journal of Computational and Applied Mathematics, vol. 233, no. 8, pp. 2027-2043, 2010.

[14] Y. Ren, A. Lin, and L. Hu, "Stochastic PDIEs and backward doubly stochastic differential equations driven by Lévy processes," Journal of Computational and Applied Mathematics, vol. 223, no. 2, pp. 901-907, 2009.

[15] S. Hamadène, "Reflected BSDE's with discontinuous barrier and application," Stochastics and Stochastics Reports, vol. 74, no. 3-4, pp. 571-596, 2002. 


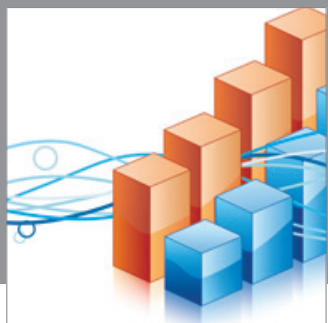

Advances in

Operations Research

mansans

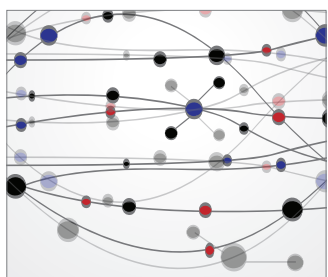

The Scientific World Journal
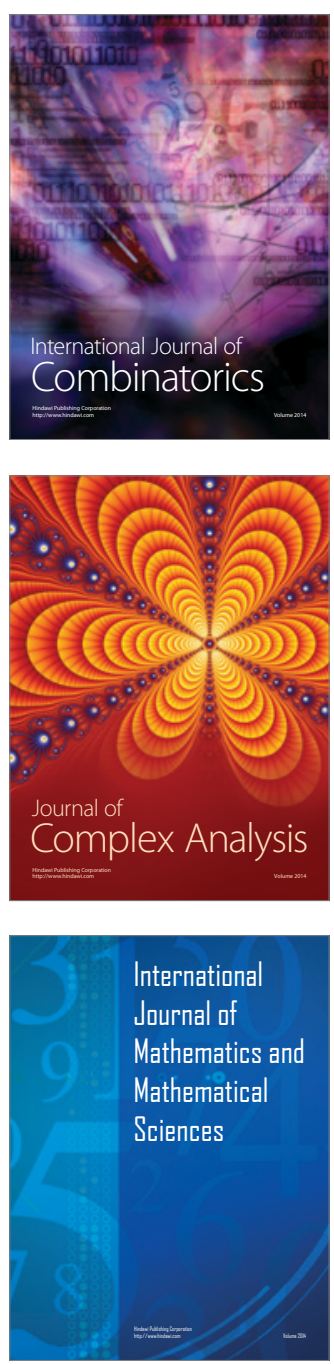
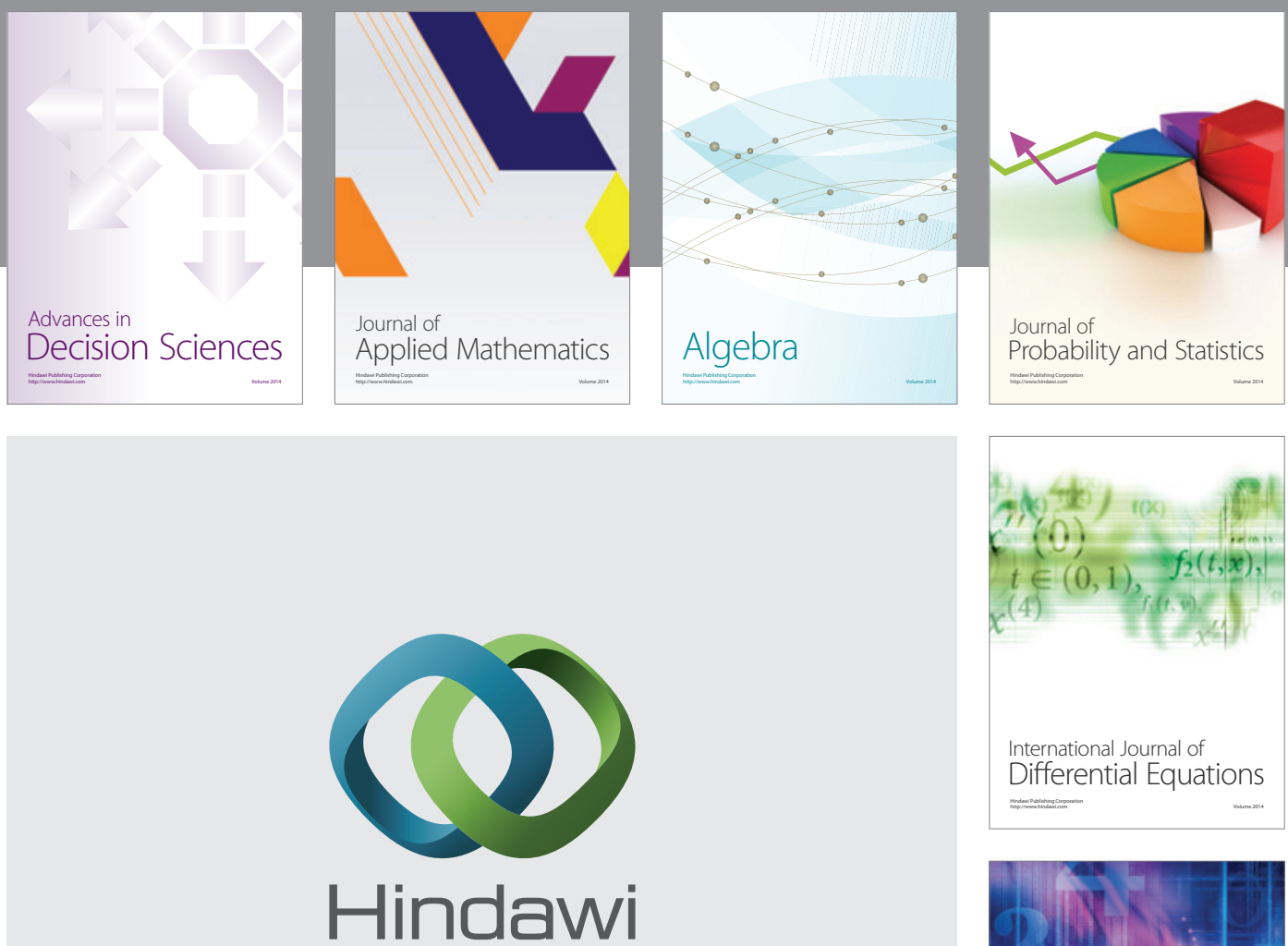

Submit your manuscripts at http://www.hindawi.com
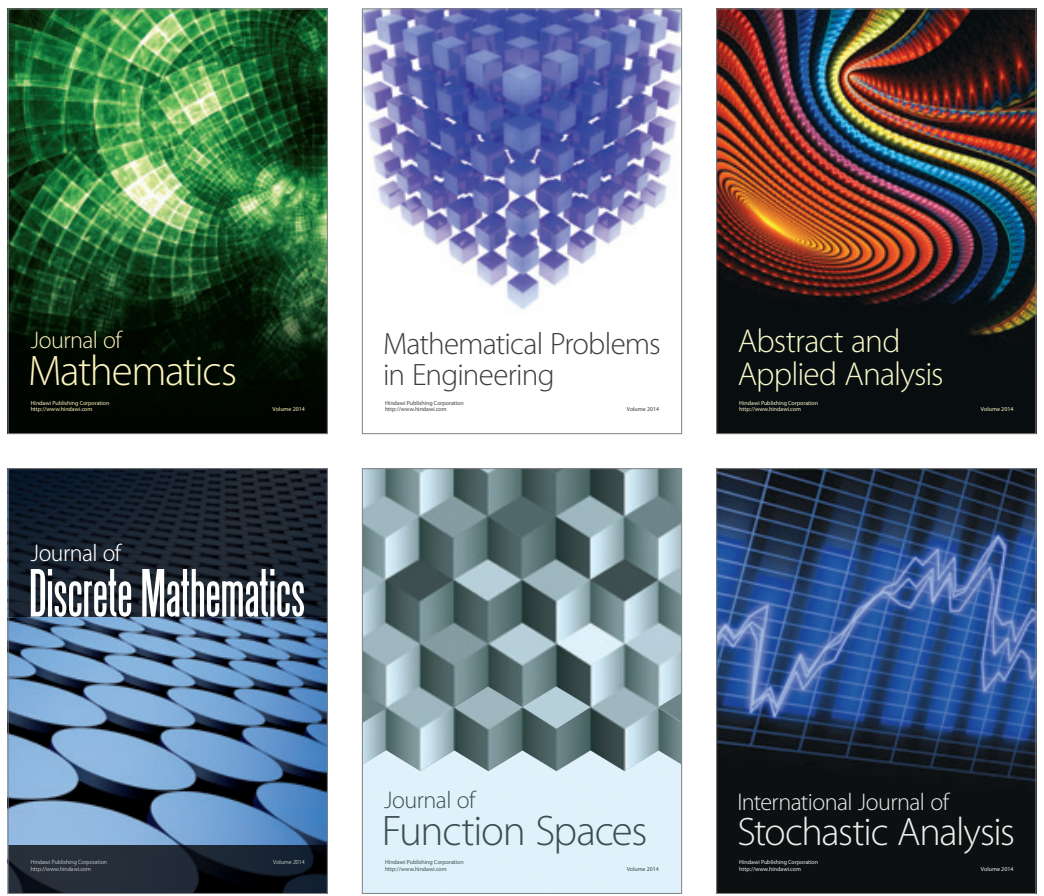

Journal of

Function Spaces

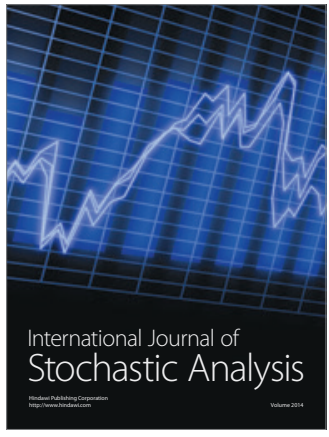

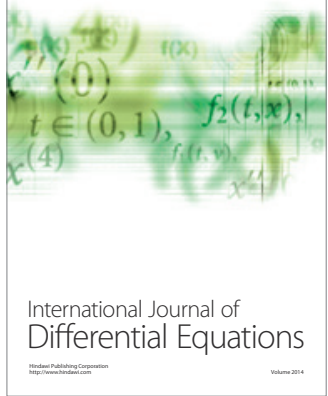
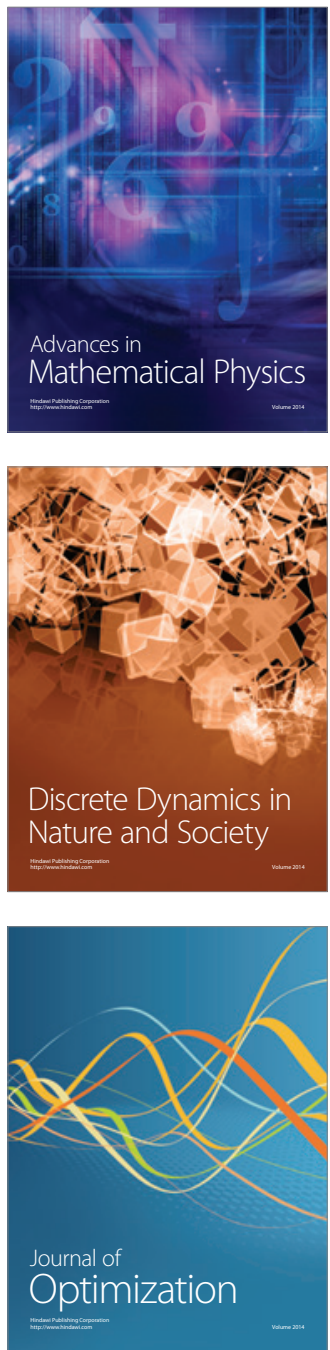